\title{
Analysis of the Problems Existing in Mechanical Structure Design of The Legged Mobile Robot
}

\author{
MingJuan Xie ${ }^{1}$, ChunYan Zhang ${ }^{1}$ and XingLu Yin ${ }^{1}$ \\ ${ }^{1}$ College of Mechanical Engineering, Shanghai University of Engineering Science ,China
}

\begin{abstract}
Based on the analysis of the research background and current situation of the legged mobile robots at home and abroad,in this paper we discusses the existing research methods of the legged mobile robot in the mechanical structure design; discusses the mainproblems in the design of the mobile robot, and to focus on the emergence of instability and poor adaptability and obstacle-surmounting performance of the legged mobile robot to find the corresponding solution in the process of movement.
\end{abstract}

\section{Introduction}

There are always some special or dangerous environments in nature, so human beings develop and use the mobile robots to engage in some dangerous environment operations. Compared with the wheeled and crawler robot, the legged mobile robot has its unique superior performance [1]: The foothold is the discrete point, which can make the robot choose the optimal support on the ground; with multi degree of freedom and adjustable leg length, the legged mobile robot keeps a stable center of gravity and balance. Therefore, in recent years, many researchers have done much research on the legged mobile robot.

However, the legged mobile robots have their own shortcomings. For example, its walking efficiency is lower and cause rollover easily due to gravity; Encountering extreme terrain will occur foot stuck, so that the entire robot lost "life." The main reason for these problems is the failure of the mechanical structure of the legged mobile robots. Therefore, the reasonable mechanical structure is the key to ensure the sufficient stability and adaptability the full function of the legged mobile robot.

\section{Research status of the legged mobile robot}

The development of the legged mobile robots begins with bipedal walking robots. In 1968, the British modeled a bipedal mobile robot $[2,3]$, the robot only has two ankles and a hip joint, the operator keeps the balance of the robot through the force feedback, but its stability and obstacle-surmounting performance are poor, so scholars at home and abroad have carried out the research on other legged mobile robot.

\subsection{One-legged mobile robot}

As shown in figure 2.1, the MIT Robot Lab produced a one-legged robot that showed jump and balance in threedimensional space in 1983,. In the process of movement, the robot achieved the stability of the jumping robot by connecting the pneumatic jumping leg and the hydraulic cylinder between the links. The mechanical structure is relatively simple and has a certain impact resistance ability $[4,5]$. A planar jump bow-legged robot developed by Carnegie Mellon University in 1999 shown in figure 2.2. It used the bow spring leg instead of pneumatic jumping leg and optimized the structure design to reduce the quality of robot and improve the robot's adaptive [6].

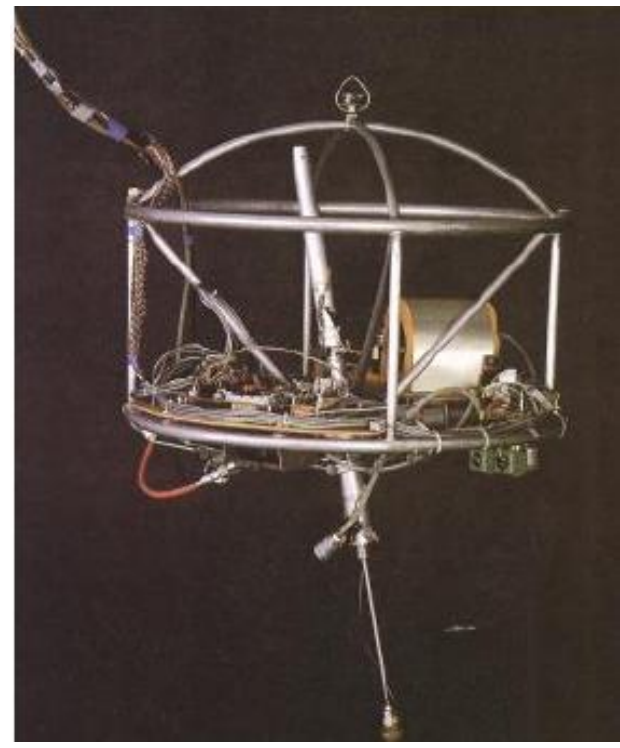

Figure 2.1. Free jumping one-legged robot physical map [4] 


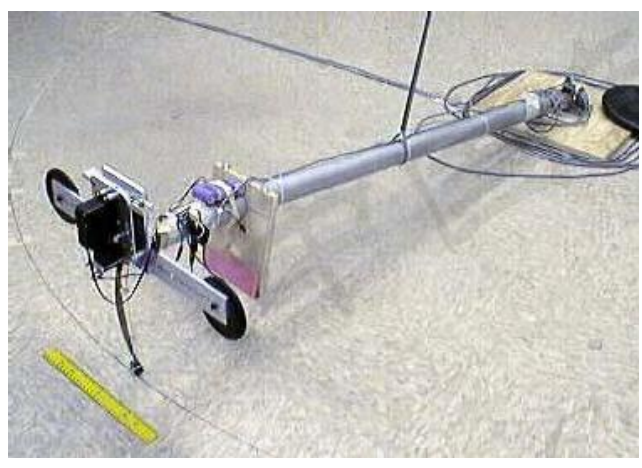

Figure 2.2. Plane jump prototype [4]

The mechanical structure of the one-legged mobile robot is relatively simple. The telescopic jump of leg can be achieved by pneumatic jumping leg or bow spring leg, and the self adaptability of the one-legged robot can be improved by adopting the bow spring leg. These mechanical structures enable the one-legged mobile robot to achieve much mechanical performance, but still have limitations in practical applications.

\subsection{Biped mobile robot}

In 1973, Kato Ichiro, of Waseda University, in Japan, developed the robot shown in figure 2.3. The robot's main body adopted multi-link mechanism to achieve the robot's static walking. Although it has the most basic components of the biped robot, but the obstaclesurmounting performance and self adaptability could not be satisfied [7].

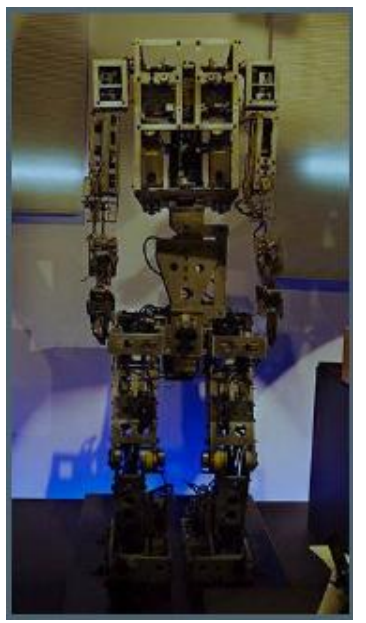

Figure 2.3. Japan Kato Ichiro bipedal robot WABOT-1 [7]

At present, there are many researches on the biped mobile robot in China. For example, Fan Shuncheng and others have analyzed the stability of the biped robot parallel leg mechanism shown in figure 2.4, each leg of the robot adopted 6-SPS parallel mechanism through the rotation and expansion of each branch and the swing and stretching movements of the legs are completed, which made the robot has good mobility and moves stably [8]. $\mathrm{Xu}$ Linsen and others made a study of biped robot walking on water mechanism and promoting mechanism, the propulsion system is the combination of Watt-I mechanism shown in figure 2.5 and the four-bar mechanism, the robot can move stably when the force on the water surface of the robot's sole does not exceed $3 \mathrm{~N}$ [9].

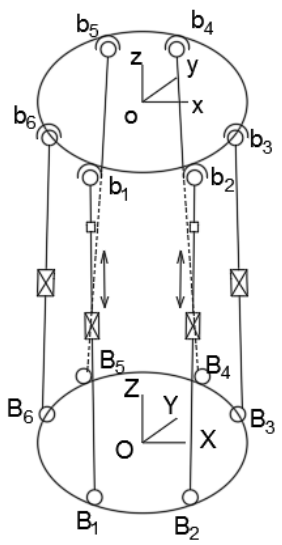

Figure 2.4. Robotic leg parallel mechanism

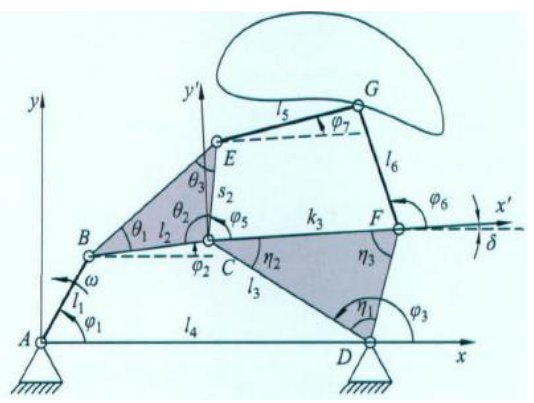

Figure 2.5. Watt-I plane mechanism [9]

Under certain conditions, these institutions can achieve the stability of the biped mobile robot, so it is necessary to consider how to enhance the stability of the robot in a wider range.

\subsection{Quadruped mobile robot}

The research of the quadruped mobile robot came into develop widely until 1980s.BigDog quadruped robot developed by the United States shown in figure 2.6. The robot was a combination of hydraulic actuator and quadruped mechanism on the whole. Each leg of the quadruped mechanism adopted a linkage mechanism, an elastic mechanism and a spring shock absorber, etc, so as to improve the flexibility and the obstacle-surmounting performance of the robot $[10,11]$.

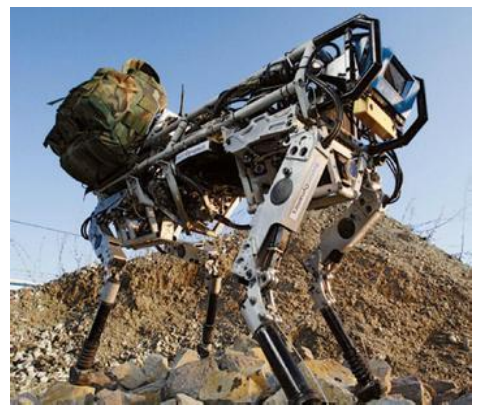

Figure 2.6. BigDog robot [11] 
In 1991, Chen Hao and others in China's Beijing University of Aeronautics and Astronautics studied on quadruped structure of the robot shown in figure 2.7, the robot's body is a pair of cross-linked structure, and the legs can be seen as a series of institutions, the middle of the two links are hinged to each other. The front end is fixed, and the rear end is respectively connected to the left and right bodies through a screw nut, which made the robot's body become wider or narrower, and improve the adaptability and obstacle-surmounting performance of the robot by controlling the nut of the screw [12].Wang Meng and others of Harbin Institute of Technology designed the frog-inspired bionic jumping robot shown in figure 2.8. The hind legs of the robot used the five-bar mechanism as the main body instead of the frog's leg multi-joint series structure, the spring instead of the muscle as the energy storage original, which make sure the stability and the obstacle-surmounting performance in the process of movement [13].

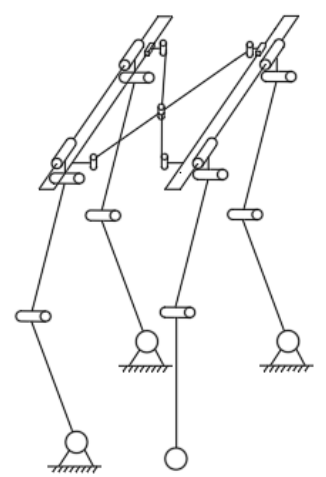

Figure 2.7. Sketch of robot mechanism

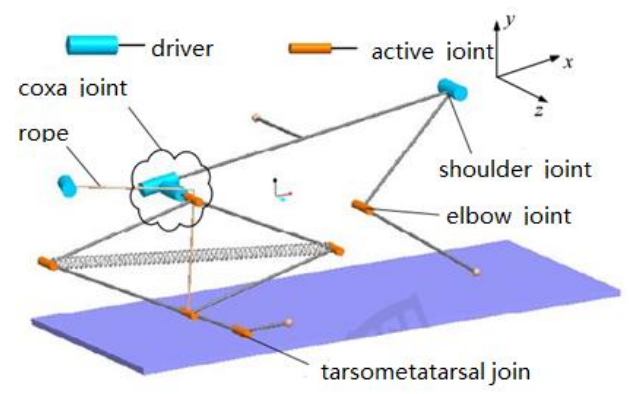

Figure 2.8. The mechanical model of frog [13]

Generally speaking, the quadruped mobile robot is superior to the biped robot in its bearing capacity and stability, and its practicability is relatively high and its technology is relatively mature.

\subsection{Six-legged mobile robot}

As shown in figure 2.9, Wang Yuefeng and others discussed the mechanical design of pantograph leg mechanism of a six-legged walking machine in 1991. The locomotion mechanism of the robot adopted a telescopic mechanism, and optimized the structure of the mechanism to enhance the stability and enlarge the movement space in the process of movement, but the obstacle-surmounting performance is poor [14]. Six- legged mobile robot has certain stability in the process of movement, but it also has many problems, such as lower flexibility or obstacle-surmounting performance or adaptability.

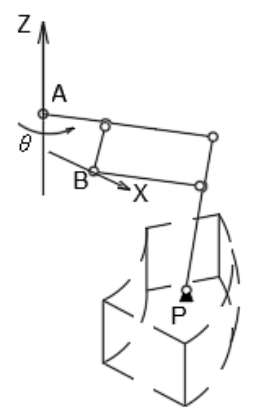

Figure 2.9.Sketch of robot leg scaling mechanism

\subsection{Eight-legged mobile robot}

Most of the eight-legged mobile robots evolved from bionic robots. At present, there are few researches on eight-legged robots at home and abroad. This paper introduces a new eight-legged gait bionic robot shown in figure 2.10 , the robot shares one motor for each pair of feet, the foot movement was caused by the gear driving the disk to rotate, and optimized the leg structure design, which can improve the obstacle-surmounting performance and self adaptability [15]. As shown in figure 2.11 , the trajectory of the D point is not only related to the length of each rod, but also to the angle of each member. The disadvantage of this mechanism is that each pair of joints on the foot has a strong coupling, which limits the flexibility of the mechanism and reduces the obstacle-surmounting performance.

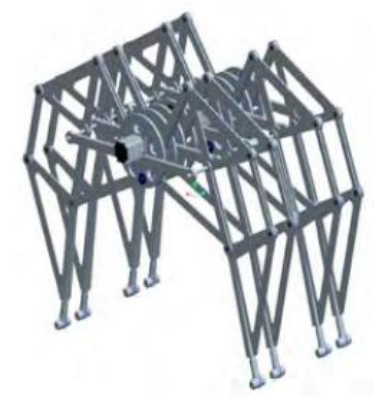

Figure 2.10. The overall model of the eight-legged bionic walking robot [15]

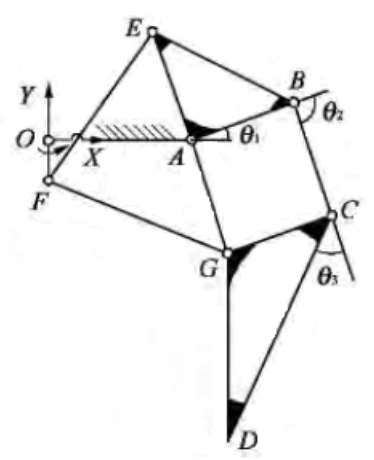

Figure 2.11. Schematic diagram of the legs [15] 
From the eight-legged mobile robot, it can be seen that the complexity of the mechanical structure will affect the flexibility and obstacle-surmounting performance of the robot.

To sum up, with the increase in the number of foot, the stability of the legged mobile robot will be improved gradually, but the mechanism has gradually become very complicate, which lead to cause some unfavorable factors, such as volume and weight increase may lead to lower flexibility of the mechanism and poor obstaclesurmounting performance. A single linkage mechanism can achieve some mechanical function, but it may not be as good as the use of the combination with a variety of mechanisms, which is beneficial to improve the overall mechanical performance of the robot. Therefore, on the basis of the existing research about the legged mobile robot in the world, aiming at summarizing the solutions of the problems in the mechanical structure design.

\section{The solution to the problems in the mechanism design of the legged mobile robot}

Many researches have been done on the key technologies of the legged mobile robots since the 1960s, and some breakthrougsent, hs have been made in some basic theoretical problems, so that the technology of the legged mobile robot has been improved continuously [16]. At preit has already formed some main research methods and theory of the legged mobile robot in the mechanical structure. After applying these research methods to practice, the stability and obstacle-surmounting performance of the robot will be improved, but they still have some problems [1]. Here are some suitable solutions of the problems that exist in the mechanical structure of legged mobile robots:

\subsection{In the process of movement,the legged mobile robot causes rollover or instability due to the center of gravity.}

In one-legged and biped mobile robots, it is common for a mobile robot to cause rollover during driving because of the center of gravity.

\subsubsection{The use of parallel mechanism to solve the problem of rollover or instability in the process of movement.}

The parallel mechanism has the characteristics of high rigidity, high precision and easy to control in real time. According to the characteristics of the parallel mechanism, the leg structure of robot can adopt 6-SPS parallel mechanism, which can complete the swing and curve movement of the leg and keep the center of gravity in the stable walking area through the rotation and expansion of each branch chain to solve the problem of rollover. Therefore, parallel mechanism is one of the ways to solve the problem of rollover.
3.1.2 A combination of various mechanisms is adopted to solve the problem of rollover or instability in the process of movement.

As shown in Fig.3.1, the four-bar mechanism, the crankrocker mechanism and the cam mechanism are used as the locomotion mechanism of the quadruped mobile robot, in which the four-bar mechanism functions as a final action, and the crank rocker mechanism is used to transmit the leg power, Cam mechanism is used to achieve the coordination of the legs of the quadruped mobile robot, thus the combination of these three mechanisms can achieve the stability of the robot [17]. Therefore, in the four-bar mechanism, pantograph mechanism, parallel mechanism and multi joint series mechanism of structure, it can be used with two or more than two simple mechanism to solve the problems of rollover or instability in the process of movement.

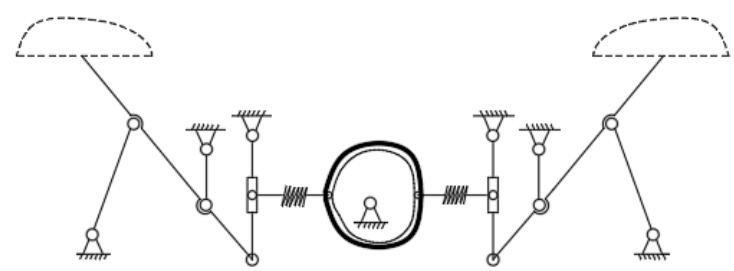

Figure 3.1. Robot half body setback mechanism

\subsection{The interference or stuck of the mechanical structures of the legged mobile robot in the process of movement.}

In the multi-legged mobile robot, with the increase of the foot, the robot's mechanism becomes complicate. At this time, it is not only necessary to optimize the design of the length of the link and the interrelation in each leg structure, but also to optimize the design of the relationship between the legs. Or it can also solve the interference or stuck problems of the mechanism by using structural singularity in reason.

\subsection{The obstacle-surmounting performance and adaptability of the legged mobile robot in the process of movement are low.}

Compared with the one-legged mobile robot, due to the complexity of the mechanical structure of the multilegged mobile robot, the obstacle-surmounting performance is lower than that of one-legged mobile robot. One-legged mobile robot generally uses a similar spring leg structure for obstacle crossing; BigDog robot also has an elastic mechanism and spring shock absorber and other structures to improve the obstacle-surmounting performance of the legged mobile robot. Therefore, an elastic mechanism may be the way to solve the problem of obstacle-surmounting performance.

As shown in Fig.3.2, the spatial pantograph mechanism acts as a branch of the parallel mechanism to control the lifting of the upper platform of the parallel mechanism, so that the mechanism can enlarge or reduce the operating space relatively [18].The most obvious feature of the pantograph mechanism is that it can reduce 
the space, and it can improve the obstacle-surmounting performance and adaptability of the mobile mechanism and achieve the overall deformation of the mobile mechanism by using the expansion and the shrinkage characteristic of the pantograph mechanism. Therefore, the pantograph mechanism can solve the problem of poor obstacle-surmounting performance and adaptability.

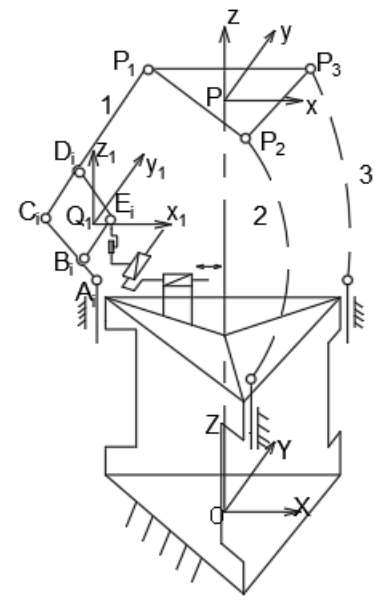

Figure 3.2. A pantograph parallel platform mechanism

\section{Conclusions}

In summary, based on the analysis of the situation and background of legged mobile robots at home and abroad, this paper analyzes and discusses the various mechanical structures of the legged mobile robots, and put forward the corresponding solution to the main problems in the design of mechanical structure. In the process of movement, the legged mobile robot can adopt the parallel mechanism or the combination of various kinds of institutions to solve the problem of rollover or instability; It can also solve the interference or stuck problems of the mechanism by optimizing the mechanical structure design and using structural singularity in reason; It can also use elastic mechanism or pantograph mechanism to solve the problem of low obstacle-surmounting performance and adaptability.

\section{References}

1. Liu Jing, Zhao Xiaoguang, Tan Ming. Legged robots A Review [J]. ROBOT, 2006,28 (1): 81-88.

2. Liu Zhiyuan. Research on Dynamic Walking of Biped Robot [D]. Harbin Institute of Technology, 1991.

3. McGhee.R.B.Robot locomotion[A].In R.Herman, S.Grillner,P.Stein,and D.Smart,editors[C].Plenum Press, 1976:237-264.

4. Su Xing. Research on Dynamic Characteristics of a
One-legged Hopping Robot Based on Pactuated Leg in Vertical Direction [D]. Harbin Institute of Technology, 2013.

5. Raibert.M.H,Brown.H.B,Jr.Chepponis.M.Experimen $\mathrm{t}$ in balance with a 3D one legged hopping machine.International J.Robotics Research. 1984(3) : 75-92.

6. Sun Zhichao. Modeling and Simulation of Onelegged Hopping Robot Using Multi-body Dynamics[D]. Harbin Institute of Technology, 2013.

7. Chen Xiaogang, Chou Zhongchen, Guan Jialiang. Development status and Prospects of Biped Robot [J]. Mechanical Engineer, 2007 (02): 17-19.

8. Fan Shuncheng, Han Shukui, Gu Huiru. The Stability of Biped Walking Robot Parallel Leg Mechanism[J].Journal of Hebei University of Technology.2004,33. (01) 27-31.

9. Xu Linsen, Mei Tao, Luo Minzhou, Huan Juan, Wei Xianming, Cao Kai. Research on Dynamical Mechanism and Propulsion System of a Biped Robot Walking on Water [J]. ROBOT, 2013,35 (03) 257262.

10. Raibert M,Blankespoor K,Nelson G,et al.BigDog,the roughterrain quadruped robot//Proceedings of the 17th International Federation of Automation Control.Seoul,2008:10822.

11. Ding Lianghong, Wang Runxiao, Feng Huashan, Li Jun.Brief Analysis of a BigDog Quadruped Robot [J]. China Mechanical Engineering, 2012,23 (05): 505514.

12. Chen Hao, Ding Xilun. Kinematics analysis of a quadruped robot with variable structure [J]. Journal of University of Science and Technology Beijing, 2012,34 (01): 53-58.

13. Wang Meng. Research on Frog-inspired Biomimetic Jumping Robot [D]. Harbin Institute of Technology.2009.

14. Wang Yuefeng, Gan Jianguo,Gan Dongying. Mechanical Design of Pantograph Legged Mechanism for a Six-legged Walking Robot [J]. Optics and Precision Engineering.1991,03:29-31.

15. Zang Hongbin, Tao Junjie. Developed of the New Eight-legged Walking Bionic Robot [J]. Journal of Mechanical Transmission.2015,39 (09): 181-185.

16. Zhu Daohong. Research of Walking Attitude Tracking Control Method of Biped Robot [D]. Xi'an University of Science And Technology, 2001.

17. Wang Jian, He Xin, Han Wang. Based on cam and crank-rocker four-legged robot gait analysis[J]. Hunan Agricultural Machinery.2011.38 (07): 60-61.

18. Zhao Tieshi, Zhao Yongsheng, Jing Wong, Liu Lechun, Huang Cheng. A Pantograph 6-dof Parallel Platform Mechanism and Its Displacement Analysis [J].ROBOT. 1998,20 (05): 346-351. 